\title{
Overall obesity, abdominal adiposity, diabetes and cigarette smoking in relation to the risk of pancreatic cancer in two Swedish population-based cohorts
}

\author{
SC Larsson*,', J Permert ${ }^{2}$, N Håkansson', I Näslund ${ }^{3}$, L Bergkvist ${ }^{4}$ and A Wolk' \\ 'Division of Nutritional Epidemiology, The National Institute of Environmental Medicine, Karolinska Institutet, Box 21 0, SE-17177 Stockholm, Sweden; \\ ${ }^{2}$ Department for Clinical Sciences, Intervention and Technology, Karolinska Institutet, Karolinska University Hospital, Huddinge, SE-I4I 86 Stockholm, \\ Sweden; ${ }^{3}$ Department of Surgery, Örebro University Hospital, SE-I 7185 Örebro, Sweden; ${ }^{4}$ Department of Surgery and Centre for Clinical Research, \\ Central Hospital, SE-72189 Västerås, Sweden
}

We examined the associations of body mass index (BMI), waist circumference, a history of diabetes, and cigarette smoking with risk of pancreatic cancer among 37 I 47 women and 45906 men followed up during 560666 person-years in the Swedish Mammography Cohort and the Cohort of Swedish Men; 136 incident cases of pancreatic cancer were diagnosed. The multivariate rate ratio (RR) of pancreatic cancer for obese women and men $\left(\mathrm{BMI} \geqslant 30 \mathrm{~kg} / \mathrm{m}^{2}\right)$ was I.8I (95\% Cl: I.04-3.15) compared to those with a BMl of $20-$ $25 \mathrm{~kg} / \mathrm{m}^{2}$. For a difference of $20 \mathrm{~cm}$ (about two standard deviations) in waist circumference, the multivariate RRs were 1.32 (95\% Cl: $0.73-2.37)$ among women and I.74 (95\% Cl: I.00-3.0 I) among men. Pancreatic cancer risk was associated with history of diabetes (multivariate RR: 1.88; 95\% Cl: 1.09-3.26) and cigarette smoking (multivariate RR for current compared with never smokers: 3.06; $95 \% \mathrm{Cl}:$ ।.99-4.72). Current smokers of $\geqslant 40$ pack-years had a five-fold elevated risk compared with never smokers. Risk among past smokers approached the RR for never smokers within 5- 10 years following smoking cessation. Findings from this prospective study support positive relationships of overall obesity, abdominal adiposity, diabetes and smoking with risk of pancreatic cancer. British Journal of Cancer (2005) 93, I310-1315. doi:I0.1038/sj.bjc.6602868 www.bjcancer.com Published online 15 November 2005 (c) 2005 Cancer Research UK

Keywords: anthropometry; body mass index; cohort studies; diabetes mellitus; pancreatic neoplasms; obesity; prospective studies; smoking; smoking cessation

Cancer of the pancreas, the sixth leading cause of cancer death in the European Union (Boyle and Ferlay, 2005), is a rapidly fatal malignancy. Less than $5 \%$ of patients survive 5 years after diagnosis (Sant et al, 2003). Primary prevention of pancreatic cancer is therefore of particular importance. Unfortunately, the etiology of pancreatic cancer remains largely elusive. Cigarette smoking is the only generally accepted modifiable risk factor, but explains only $25-29 \%$ of pancreatic cancer incidence (Silverman et al, 1994; Fuchs et al, 1996).

Evidence from in vitro, animal and human studies indicates that insulin, insulin resistance and abnormal glucose metabolism may play a role in pancreatic cancer etiology (Fisher et al, 1996; Gapstur et al, 2000; Schneider et al, 2001; Jee et al, 2005). Moreover, epidemiological studies have suggested a relationship between diabetes mellitus and increased risk of pancreatic cancer (Huxley et al, 2005). Obesity, and specifically abdominal adiposity, has been linked to metabolic abnormalities, including insulin resistance, hyperinsulinemia, glucose intolerance and to the development of diabetes mellitus (Kahn and Flier, 2000; IARC, 2002). Thus, obesity may be a risk factor for pancreatic cancer. However,

*Correspondence: Dr SC Larsson; E-mail: susanna.larsson@ki.se Received 24 August 2005; revised 10 October 2005; accepted 17 October 2005; published online 15 November 2005 epidemiological studies of body mass index (BMI), as a measure of overall obesity, in relation to risk of pancreatic cancer have yielded inconsistent results with a positive association observed in some, but not all studies (Berrington de Gonzalez et al, 2003). To our knowledge, no study has investigated the association between waist circumference, as an estimate of abdominal adiposity, and risk of pancreatic cancer.

In two prospective population-based cohorts of Swedish women and men, we examined the relationships between overall obesity (reflected by BMI) and abdominal adiposity (waist circumference) and the risk of pancreatic cancer. In addition, we report findings for diabetes, cigarette smoking and smoking cessation on pancreatic cancer risk.

\section{MATERIALS AND METHODS}

\section{Study population}

Two population-based prospective cohort studies provided data for the present analyses: the Swedish Mammography Cohort (SMC) and the Cohort of Swedish Men (COSM). The SMC was established between 1987 and 1990, when all women born between 1914 and 1948 and living in central Sweden (Västmanland and Uppsala counties) received a mailed questionnaire that elicited 
information on diet, weight, height and education; 66651 women (74\% of the source population) returned a completed questionnaire. In the autumn of 1997, an expanded questionnaire that included data on various lifestyle factors and medical history was mailed to women who were still alive and residing in the study area; 39227 women $(70 \%)$ completed the questionnaire. In the autumn of 1997, 48850 men born between 1918 and 1952 and residing in central Sweden (Västmanland and Örebro counties) were enrolled in the COSM by return of a mailed questionnaire that was identical to the 1997 SMC questionnaire (except for some sex-specific questions).

Eligible participants for the present study were women and men who completed the 1997 questionnaire (information on cigarette smoking and diabetes was first obtained in 1997). After excluding participants with erroneous or missing national registration number and those with a cancer diagnosis (except nonmelanoma skin cancer) before baseline, a total of 37147 women and 45906 men were eligible for follow-up. The study was approved by the Regional Ethical Review Board in Stockholm.

\section{Assessment of exposures}

In 1997, participants completed a self-administered questionnaire that included information on demographic characteristics, weight, height, waist circumference, cigarette smoking status and history, alcohol consumption, dietary intake, physical activity and history of diabetes mellitus; complementary information on diabetes for subjects who were hospitalized was obtained from the Swedish Inpatient Register. Pack-years were estimated from baseline smoking history by multiplying the number of years of smoking by the average number of packs of cigarettes smoked. We estimated BMI from self-reported weight and height $(\mathrm{kg} / \mathrm{height}$ in $\mathrm{m}^{2}$ ) as a measure of overall obesity. High validity has been observed for self-reported height $(r=0.9)$ and weight $(r=0.9)$ compared with actual measurement among Swedish women and men (Kuskowska-Wolk et al, 1989). We used self-reported waist circumference as an estimate of abdominal adiposity. We do not have validity data for self-report of waist from the Swedish population, but results from an UK population have indicated high validity $(r=0.8)$ (Spencer et al, 2004).

\section{Ascertainment of cases and follow-up}

Incident cases of pancreatic cancer were identified by computerised record linkage of the study population to the National Swedish Cancer Register and the Regional Cancer Register covering the study area, both of which have been estimated to be nearly 100\% complete (Mattsson and Wallgren, 1984). Pancreatic cancer cases were defined as primary malignant neoplasm of the exocrine pancreas [International Classification of Diseases, Ninth Revision (ICD-9) code 157]. We excluded islet-cell carcinomas (ICD-9 code 157.4) because the etiology of these tumors may be different from that of the exocrine pancreas. Information on dates of death and dates of moving out of the study area was obtained from the Swedish Death and Population registries.

\section{Statistical analysis}

Person-years of follow-up for each participant were computed as the time from baseline to the date of diagnosis of pancreatic cancer, death from any cause, emigration, or until December 31, 2004. Participants were categorized into four groups with BMI $\left(\mathrm{kg} / \mathrm{m}^{2}\right)$ corresponding to $<20.0,20.0-24.9$ (reference group), $25.0-29.9$ and $\geqslant 30.0$. Participants with a BMI below 15 or above $60 \mathrm{~kg} / \mathrm{m}^{2}$ were excluded in the BMI analyses. For waist circumference, we used quartiles based on the distribution among women and men separately.
Cox proportional hazards models (Cox and Oakes, 1984) were used to estimate rate ratios (RRs) with $95 \%$ confidence intervals (CIs). Age (in months) and sex were controlled for as stratification variables in the Cox model. In multivariate models, we included education (less than high school, high-school graduate, or more than high school), BMI $\left(<20.0,20.0-25.0,25.0-29.9\right.$, or $\left.\geqslant 30 \mathrm{~kg} / \mathrm{m}^{2}\right)$, physical activity (four categories), history of diabetes (yes or no), cigarette smoking (never, past $<20$ pack-years, past $\geqslant 20$ packyears, current $<20$ pack-years, current 20-39 pack-years, or current $\geqslant 40$ pack-years) and alcohol consumption (quartiles). Multivariate analyses of waist circumference were further controlled for height (quartiles). Trends tests for BMI and waist circumference were performed by scoring the categories and entering the score as a continuous term in the regression model. Statistical interaction between BMI and cigarette smoking in relation to pancreatic cancer risk was tested using the likelihood ratio test. We used the SAS statistical package (version 9.1; SAS Institute, Inc., Cary, NC, USA) for all analyses. All statistical tests are two-sided.

\section{RESULTS}

A total of 136 incident cases (61 females and 75 males) of pancreatic cancer were diagnosed during 560666 person-years of follow-up, yielding a crude incidence rate of 24.3 per 100000 person-years. The incidence rate was similar among women (24.1 per 100000 person-years) and men (24.4 per 100000 personyears). The mean ( \pm s.d.) age of the participants at baseline in 1997 was 62 (9.3) years for women and 60 (9.7) years for men. The mean ( \pm s.d.) age of the participants at time of diagnosis of pancreatic cancer was 72 (8.5) years for female cases and 72 (8.0) years for male cases. Table 1 presents the baseline characteristics by BMI. The mean ( \pm s.d.) baseline BMI was 25.0 (3.9) $\mathrm{kg} / \mathrm{m}^{2}$ among women and $25.8(3.4) \mathrm{kg} / \mathrm{m}^{2}$ among men. In all, $34 \%$ percent of the women and $46 \%$ of the men were overweight (BMI $25-29.9 \mathrm{~kg} / \mathrm{m}^{2}$ ); $11 \%$ percent of the women and $10 \%$ of the men were obese $\left(B M I \geqslant 30 \mathrm{~kg} / \mathrm{m}^{2}\right)$. Compared with women and men with a BMI of $20-25 \mathrm{~kg} / \mathrm{m}^{2}$, obese women and men were less likely to have a post-secondary education and to be current smokers, but were more likely to have diabetes; they also exercised less and had lower alcohol consumption. BMI was strongly correlated with waist circumference (women, $r=0.76$; men, $r=0.75$ ). The proportions of never, past and current smokers, respectively, at baseline were 54, 27 and 19\% among women and 36, 46 and $18 \%$ among men.

We observed a positive association between BMI and risk of pancreatic cancer (Table 2). After controlling for age and other potential risk factors for pancreatic cancer, women and men with a BMI of $30 \mathrm{~kg} / \mathrm{m}^{2}$ or higher had an $81 \%$ increased risk compared with those with a BMI of $20-25 \mathrm{~kg} / \mathrm{m}^{2}$. When analysed as a continuous variable, an increment of 1 BMI unit $\left(\mathrm{kg} / \mathrm{m}^{2}\right)$ was related to a $5 \%$ increased risk of pancreatic cancer among women and men combined (RR: $1.05 ; 95 \%$ CI: $1.00-1.10$ ), a $4 \%$ increased risk among women (RR: 1.04; 95\% CI: $0.97-11$ ), and a $6 \%$ increased risk among men (RR: 1.06; 95\% CI: 0.99-1.14). The findings for BMI did not change appreciably after excluding cases that occurred within the first year of follow-up. Waist circumference was also positively associated with risk of pancreatic cancer, and similar to the observed association for BMI; the risk was greater among men (Table 2). For a difference of $20 \mathrm{~cm}$ (approximately two standard deviations) in waist circumference, the multivariate RRs were 1.32 (95\% CI: $0.73-2.37)$ among women and 1.74 (95\% CI: $1.00-3.01)$ among men.

A history of diabetes was associated with a statistically significant 1.9-fold elevated risk of pancreatic cancer (Table 2). This association remained after early follow-up cancers (i.e., those diagnosed within the first year) were excluded (RR: 1.97; $95 \%$ 
Table I Age-standardised characteristics among women and men according to categories of body mass index at baseline

Body mass index $\left(\mathrm{kg} / \mathrm{m}^{2}\right)$

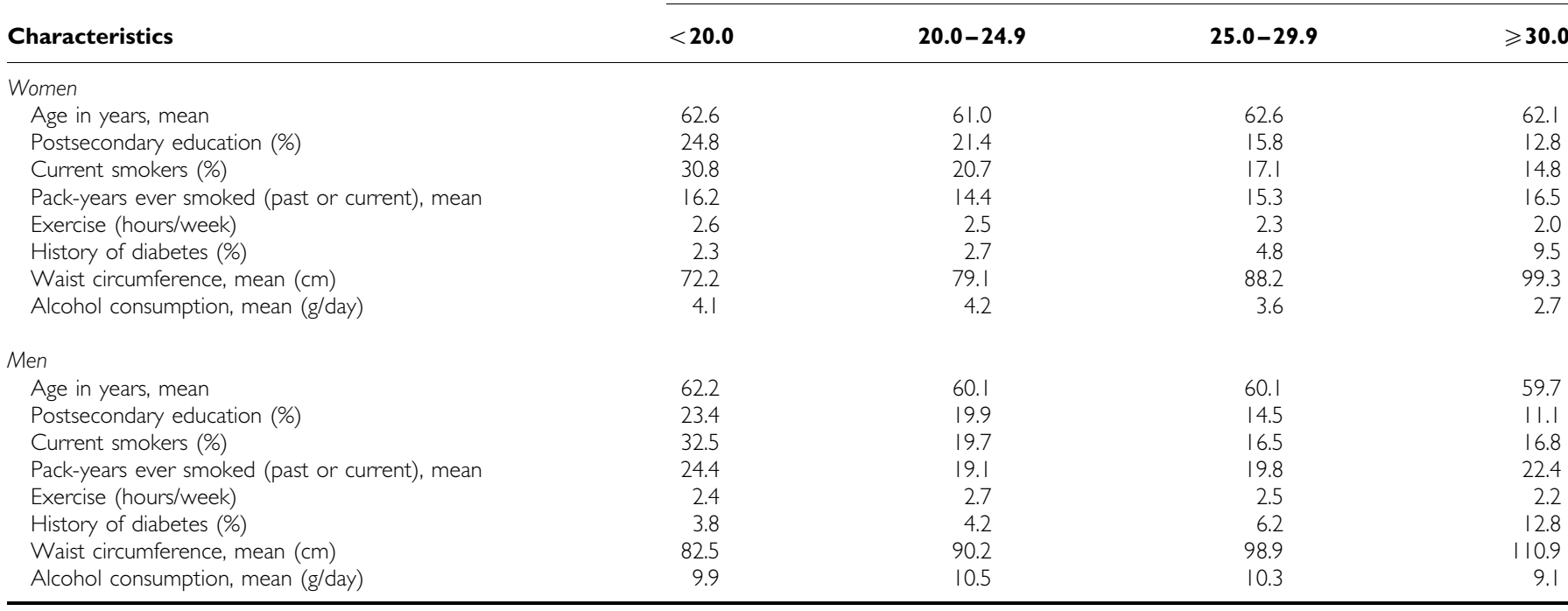

Table 2 Rate ratios (95\% confidence intervals) of pancreatic according to body mass index, waist circumference, and diabetes

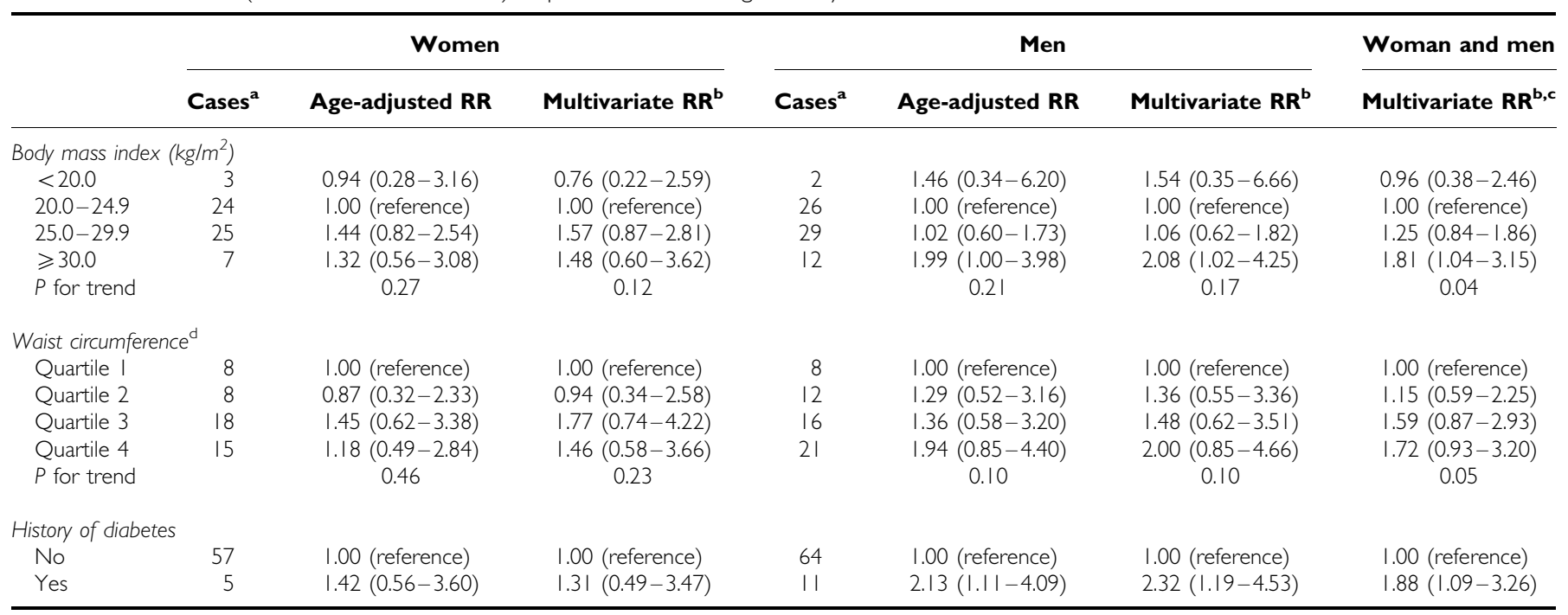

The number of cases may not sum up to the total number of cases owing to missing data on BMl or waist circumference. ${ }^{\mathrm{b}}$ Multivariate models were stratified by age in months at baseline and were simultaneously adjusted for education (less than high school, high school graduate, and more than high school), physical activity (four categories), cigarette smoking (never, past $<20$ pack-years, past $\geqslant 20$ pack-years, current $<20$ pack-years, current 20-39 pack-years, or current $\geqslant 40$ pack-years), and alcohol consumption (quartiles). Analyses of body mass index were further adjusted for diabetes; analyses of waist circumference were further adjusted for diabetes and height (quartiles); analyses of diabetes were further adjusted for body mass index. 'Also adjusted for sex. ${ }^{\mathrm{d} C u t}$ points for quartiles of waist circumference (cm) for women were $<76,76-81,82-89$, and $\geqslant 90$; for men $<90,90-94,95-101$, and $\geqslant 102$

CI: $1.10-3.53)$. The association with diabetes seemed to be confined to men, but the number of diabetic women with pancreatic cancer was small $(n=5)$.

Women and men who were current smokers at baseline had a significantly higher risk of pancreatic cancer compared with never smokers (Table 3 ). Past smoking was not related to a significantly increased risk, although women who had smoked in the past had a nonsignificant elevation in risk. Risk of pancreatic cancer increased with increasing number of pack-years of smoking. Current smokers of 40 or more pack-years had a fivefold elevated risk of pancreatic cancer compared to never smokers. An increment of 10 pack-years of smoking was associated with a multivariate $\mathrm{RR}$ of $1.36(95 \% \mathrm{CI}: 1.15-1.61 ; P$ value for trend $=0.0003)$.

We also assessed the association between smoking cessation and risk of pancreatic cancer (Figure 1). Compared with current smokers, the RR among past smokers diminished steeply and approached the RR for never smokers within 5-10 years following smoking cessation.

Figure 2 shows the RRs of pancreatic cancer according to crossclassification of BMI and smoking status. High BMI and cigarette smoking were independently associated with increased risk of 
Table 3 Rate ratios (95\% confidence intervals) of pancreatic cancer according to cigarette smoking status and total number of pack-years of smoking

\begin{tabular}{|c|c|c|c|c|c|c|c|}
\hline & \multicolumn{3}{|c|}{ Women } & \multicolumn{3}{|c|}{ Men } & $\frac{\text { Woman and men }}{\text { Multivariate } \mathbf{R R}^{\mathrm{a}, \mathrm{b}}}$ \\
\hline \multicolumn{8}{|l|}{ Smoking status } \\
\hline Never smoked & 26 & I.00 (reference) & 1.00 (reference) & 22 & 1.00 (reference) & 1.00 (reference) & 1.00 (reference) \\
\hline Past smoker & 13 & $1.48(0.75-2.93)$ & $1.39(0.69-2.78)$ & 32 & $1.10(0.64-1.89)$ & $1.00(0.57-1.74)$ & $1.16(0.75-1.80)$ \\
\hline Current smoker & 22 & $3.44(1.91-6.19)$ & $3.81(2.08-7.00)$ & 21 & $2.52(1.38-4.63)$ & $2.47(1.33-4.55)$ & $3.06(1.99-4.72)$ \\
\hline \multicolumn{8}{|c|}{ Pack-years, ${ }^{c}$ past smokers } \\
\hline$<20$ & 7 & $0.95(0.41-2.22)$ & $0.90(0.38-2.12)$ & 18 & $0.90(0.48-1.69)$ & $0.84(0.44-1.58)$ & $0.92(0.56-1.53)$ \\
\hline$\geqslant 20$ & 6 & $4.36(1.75-10.89)$ & $4.15(1.63-10.5 \mid)$ & 14 & $1.52(0.77-2.98)$ & $1.33(0.67-2.66)$ & $1.91(1.08-3.35)$ \\
\hline \multicolumn{8}{|c|}{ Pack-years, ${ }^{c}$ current smokers } \\
\hline Never smoked & 26 & I.00 (reference) & ।.00 (reference) & 22 & I.00 (reference) & 1.00 (reference) & 1.00 (reference) \\
\hline$<20$ & 8 & $2.38(1.06-5.34)$ & $2.65(1.16-6.05)$ & 7 & $2.33(0.98-5.53)$ & $2.30(0.97-5.50)$ & $2.46(1.35-4.49)$ \\
\hline $20-39$ & II & $4.21(2.02-8.78)$ & $4.81(2.27-10.18)$ & 5 & $1.54(0.58-4.13)$ & $1.53(0.57-4.11)$ & $2.91(1.62-5.25)$ \\
\hline
\end{tabular}

aMultivariate models were stratified by age in months at baseline and were simultaneously adjusted for education (less than high school, high school graduate, and more than high school), body mass index (<20.0, 20.0-24.9, $25.0-29.9$, or $\geqslant 30 \mathrm{~kg} / \mathrm{m}^{2}$ ), physical activity (four categories), history of diabetes (yes, no), and alcohol consumption (quartiles).

${ }^{\mathrm{b}}$ Also adjusted for sex. ${ }^{C}$ Number of packs of cigarettes smoked per day (one pack contains 20 cigarettes) multiplied by the number of years of smoking.

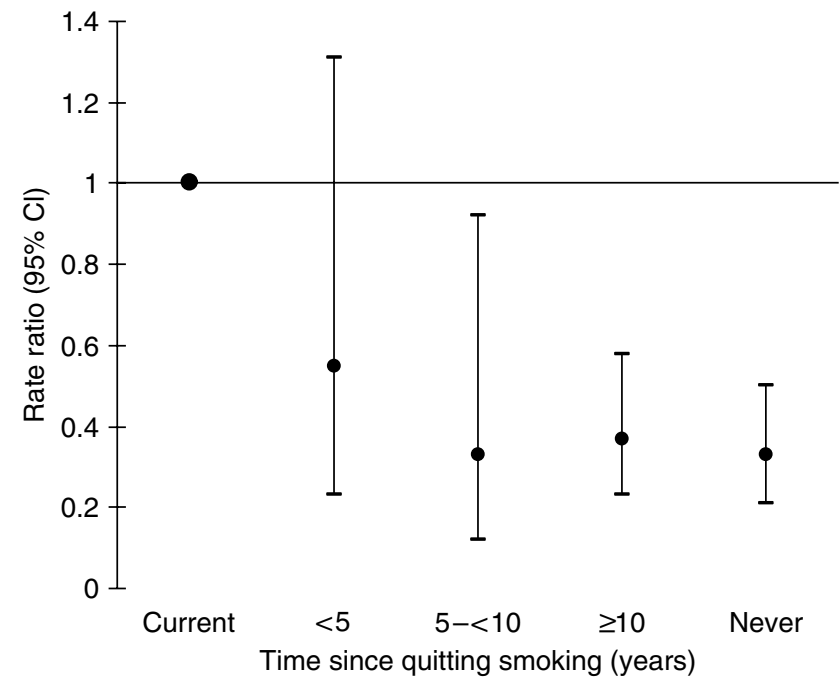

Figure I Multivariate rate ratios of pancreatic cancer in relation to time since quitting of smoking among women and men. Multivariate models were stratified by age in months at baseline and sex and were simultaneously adjusted for education (less than high school, high school graduate, or more than high school), body mass index (<20.0, 20.0-24.9, $25.0-29.9$, or $\geqslant 30 \mathrm{~kg} / \mathrm{m}^{2}$ ), physical activity (four categories), history of diabetes (yes or no), and alcohol consumption (quartiles).

pancreatic cancer and there was no apparent effect modification between these variables $(P$ for interaction $=0.74)$. Compared with nonsmokers (never and past) with a BMI of less than $25 \mathrm{~kg} / \mathrm{m}^{2}$, current smokers with a BMI of $30 \mathrm{~kg} / \mathrm{m}^{2}$ or more, had a multivariate RR of 5.07 (CI: $1.93-13.30$ ).

\section{DISCUSSION}

In this prospective analysis, we observed approximately a doubling of the risk of pancreatic cancer for obese women and men (BMI $\geqslant 30 \mathrm{~kg} / \mathrm{m}^{2}$ ) compared with those with a normal weight (BMI $20-25 \mathrm{~kg} / \mathrm{m}^{2}$ ). We also confirmed associations between history of

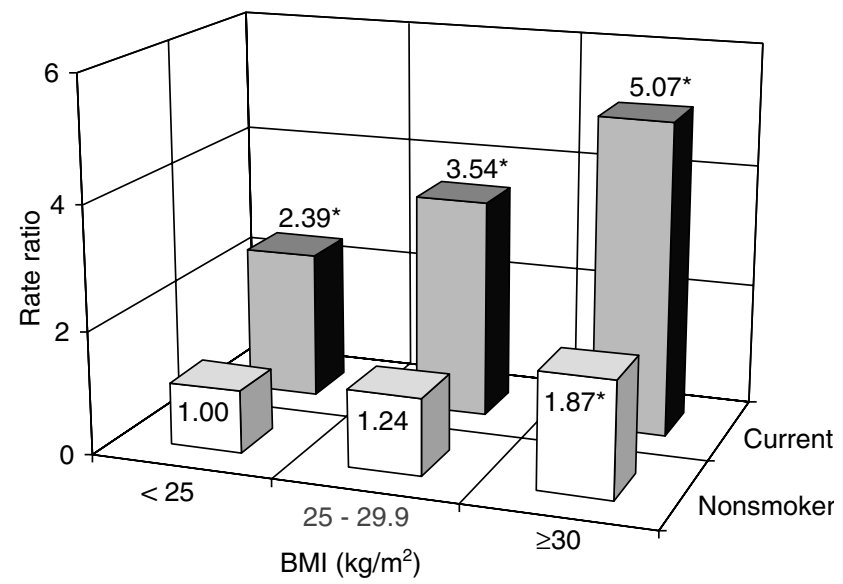

Figure 2 Multivariate rate ratios of pancreatic cancer according to body mass index (BMI) and cigarette smoking status among women and men. Multivariate models were stratified by age in months at baseline and sex and were simultaneously adjusted for education (less than high school, high school graduate, or more than high school), physical activity (four categories), history of diabetes (yes or no), and alcohol consumption (quartiles). Nonsmoker include never and past smokers. *Statistically significant.

diabetes and cigarette smoking and increased risk of pancreatic cancer. In addition, we found that smokers who had quit smoking for 5-10 years had a risk of developing pancreatic cancer similar to that of never smokers.

To our knowledge, this was the first study to evaluate the relationship between waist circumference (reflecting abdominal adiposity) and risk of pancreatic cancer. We found that waist circumference was positively associated with pancreatic cancer risk, particularly among men. A previous cohort study found that women and men who reported 'central' weight gain had increased relative risk of pancreatic cancer compared with women and men who reported peripheral weight gain; the association was stronger among men (Patel et al, 2005).

Previous studies of BMI and pancreatic cancer risk have produced inconsistent results. A recent meta-analysis of 14 studies 
on obesity and risk of pancreatic cancer estimated a 19\% increase in risk among obese individuals compared with those with a normal weight (RR: 1.19; 95\% CI: $1.10-1.29$ for BMI $30 v s$ $22 \mathrm{~kg} / \mathrm{m}^{2}$ ) (Berrington de Gonzalez et al, 2003). The summary risk estimate was higher when the authors excluded studies that did not control for smoking; the estimate was also higher for cohort than for case-control studies (Berrington de Gonzalez et al, 2003). Since that meta-analysis, two case-control studies based on direct patient interviews (Pan et al, 2004; Fryzek et al, 2005) and two cohort studies (Samanic et al, 2004; Patel et al, 2005) have reported a statistically significant positive relation between BMI and pancreatic cancer risk in both women and men or only in men. Another cohort of elderly women observed no increase in pancreatic cancer risk with greater BMI (Sinner et al, 2005).

In the present study, we observed a 1.9-fold higher risk of pancreatic cancer among women and men with diabetes. A metaanalysis of 17 case-control and 19 cohort or nested case-control studies (Huxley et al, 2005) showed a 1.8-fold higher risk of pancreatic cancer associated with a history of diabetes; the magnitude of the positive association was greater among individuals whose diabetes had been recently diagnosed.

A metabolic consequence of obesity, particularly the accumulation of intra-abdominal fat, is the development of insulin resistance, which leads to an increase in the secretion of insulin from the pancreas (IARC, 2002). A role of hyperinsulinemia in pancreatic pathogenesis might be through an increase in local blood flow and cell division in the pancreas (Henderson et al, 1981). The exocrine pancreatic cells are exposed to very high insulin concentrations through a portal circulation system from the insulin-producing pancreatic islets (Williams and Goldfine, 1985). High concentrations of insulin are able to activate the insulin-like growth factor I (IGF-I) receptor, and activation of this receptor leads to growth-promoting effects (Le Roith, 1997). Furthermore, excess insulin, through downregulation of the insulin-like growth factor binding protein-1, could result in an increase in the exposure to free IGF-I (Giovannucci, 2003). Both insulin and IGF-I have been shown to promote growth in pancreatic cell lines (Ohmura et al, 1990; Takeda and Escribano, 1991; Bergmann et al, 1995; Fisher et al, 1996).

This study has several strengths. The prospective design precluded recall bias and the need to use next-of-kin respondents. In addition, we had virtually complete follow-up as incident cases of pancreatic cancer were ascertained by record linkage to the Swedish Cancer Registry. Differential follow-up is therefore unlikely to have affected our results. We were also able to control for potential confounding by most known or possible risk factors for pancreatic cancer. Because data regarding exposures were collected before the diagnosis of pancreatic cancer, any misclassification would be nondifferential and would most likely have weakened rather than exaggerated any true associations. A limitation of this study is the self-reported information on anthropometric measures, diabetes and cigarette smoking. Overweight and obese individuals tend to underestimate their body size (weight, BMI, waist) to a greater extent than those who are lean, and shorter individuals tend to overestimate their height (Kuskowska-Wolk et al, 1989; Spencer et al, 2004). These biases are likely to attenuate risk estimates of the relations between BMI and waist circumference and risk of pancreatic cancer; thus the associations may be even stronger in the absence of measurement error. Another limitation of our study is the lack of information on timing of diagnosis of diabetes.

In summary, results from this population-based prospective study suggest that obesity may increase the risk for pancreatic cancer. Findings from this study also provide further support for associations of history of diabetes and cigarette smoking with pancreatic cancer risk.

\section{ACKNOWLEDGEMENTS}

This work was supported by research grants from the Swedish Research Council/Longitudinal Studies, the Swedish Cancer Foundation, Västmanland County Research Fund against Cancer, Örebro County Council Research Committee and Örebro Medical Center Research Foundation.

\section{REFERENCES}

Bergmann U, Funatomi H, Yokoyama M, Beger HG, Korc M (1995) Insulinlike growth factor I overexpression in human pancreatic cancer: evidence for autocrine and paracrine roles. Cancer Res 55: 2007-2011

Berrington de Gonzalez A, Sweetland S, Spencer E (2003) A metaanalysis of obesity and the risk of pancreatic cancer. $B r J$ Cancer 89: $519-523$

Boyle P, Ferlay J (2005) Cancer incidence and mortality in Europe, 2004. Ann Oncol 16: $481-488$

Cox DR, Oakes D (1984) Analysis of Survival Data. London: Chapman \& Hall

Fisher WE, Boros LG, Schirmer WJ (1996) Insulin promotes pancreatic cancer: evidence for endocrine influence on exocrine pancreatic tumors. J Surg Res 63: $310-313$

Fryzek JP, Schenk M, Kinnard M, Greenson JK, Garabrant DH (2005) The association of body mass index and pancreatic cancer in residents of Southeastern Michigan, 1996-1999. Am J Epidemiol 162: $222-228$

Fuchs CS, Colditz GA, Stampfer MJ, Giovannucci EL, Hunter DJ, Rimm EB, Willett WC, Speizer FE (1996) A prospective study of cigarette smoking and the risk of pancreatic cancer. Arch Intern Med 156: 2255-2260

Gapstur SM, Gann PH, Lowe W, Liu K, Colangelo L, Dyer A (2000) Abnormal glucose metabolism and pancreatic cancer mortality. JAMA 283: $2552-2558$

Giovannucci E (2003) Nutrition, insulin, insulin-like growth factors and cancer. Horm Metab Res 35: 694-704

Henderson JR, Daniel PM, Fraser PA (1981) The pancreas as a single organ: the influence of the endocrine upon the exocrine part of the gland. Gut 22: $158-167$
Huxley R, Ansary-Moghaddam A, Berrington de Gonzalez A, Barzi F, Woodward M (2005) Type-II diabetes and pancreatic cancer: a metaanalysis of 36 studies. Br J Cancer 92: 2076-2083

IARC (2002) IARC Handbooks of Cancer Prevention. Weight Control and Physical Activity Vol. 6. Lyon, France: IARC Press

Jee SH, Ohrr H, Sull JW, Yun JE, Ji M, Samet JM (2005) Fasting serum glucose level and cancer risk in Korean men and women. JAMA 293: $194-202$

Kahn BB, Flier JS (2000) Obesity and insulin resistance. J Clin Invest 106: $473-481$

Kuskowska-Wolk A, Karlsson P, Stolt M, Rossner S (1989) The predictive validity of body mass index based on self-reported weight and height. Int J Obes 13: $441-453$

Le Roith D (1997) Seminars in medicine of the Beth Israel Deaconess Medical Center. Insulin-like growth factors. $N$ Engl J Med 336: 633-640

Mattsson B, Wallgren A (1984) Completeness of the Swedish Cancer Register. Non-notified cancer cases recorded on death certificates in 1978. Acta Radiol Oncol 23: 305-313

Ohmura E, Okada M, Onoda N, Kamiya Y, Murakami H, Tsushima T, Shizume K (1990) Insulin-like growth factor I and transforming growth factor alpha as autocrine growth factors in human pancreatic cancer cell growth. Cancer Res 50: 103-107

Pan SY, Johnson KC, Ugnat AM, Wen SW, Mao Y (2004) Association of obesity and cancer risk in Canada. Am J Epidemiol 159: 259-268

Patel AV, Rodriguez C, Bernstein L, Chao A, Thun MJ, Calle EE (2005) Obesity, recreational physical activity, and risk of pancreatic cancer in a large U.S. Cohort. Cancer Epidemiol Biomarkers Prev 14: 459-466 
Samanic C, Gridley G, Chow WH, Lubin J, Hoover RN, Fraumeni Jr JF (2004) Obesity and cancer risk among white and black United States veterans. Cancer Causes Control 15: 35-43

Sant M, Aareleid T, Berrino F, Bielska Lasota M, Carli PM, Faivre J, Grosclaude P, Hedelin G, Matsuda T, Moller H, Moller T, Verdecchia A, Capocaccia R, Gatta G, Micheli A, Santaquilani M, Roazzi P, Lisi D (2003) EUROCARE-3: survival of cancer patients diagnosed 1990-94 - results and commentary. Ann Oncol 14(Suppl 5): v61-v118

Schneider MB, Matsuzaki H, Haorah J, Ulrich A, Standop J, Ding XZ, Adrian TE, Pour PM (2001) Prevention of pancreatic cancer induction in hamsters by metformin. Gastroenterology 120: 1263-1270

Silverman DT, Dunn JA, Hoover RN, Schiffman M, Lillemoe KD, Schoenberg JB, Brown LM, Greenberg RS, Hayes RB, Swanson GM
(1994) Cigarette smoking and pancreas cancer: a case-control study based on direct interviews. J Natl Cancer Inst 86: 1510-1516

Sinner PJ, Schmitz KH, Anderson KE, Folsom AR (2005) Lack of association of physical activity and obesity with incident pancreatic cancer in elderly women. Cancer Epidemiol Biomarkers Prev 14: 1571 - 1573

Spencer EA, Roddam AW, Key TJ (2004) Accuracy of self-reported waist and hip measurements in 4492 EPIC-Oxford participants. Public Health Nutr 7: 723-727

Takeda Y, Escribano MJ (1991) Effects of insulin and somatostatin on the growth and the colony formation of two human pancreatic cancer cell lines. J Cancer Res Clin Oncol 117: 416-420

Williams JA, Goldfine ID (1985) The insulin-pancreatic acinar axis. Diabetes 34: 980 -986 